NISTIR 7457

\title{
Recommendations and Guidelines for Standardization of Fluorescence Spectroscopy
}

\author{
Paul C. DeRose \\ Biochemical Science Division \\ National Institute of Standards and Technology \\ Gaithersburg, MD 20899-8312
}


NISTIR 7457

\title{
Recommendations and Guidelines for Standardization of Fluorescence Spectroscopy
}

\author{
Paul C. DeRose \\ Biochemical Science Division \\ National Institute of Standards and Technology \\ Gaithersburg, MD 20899-8312
}

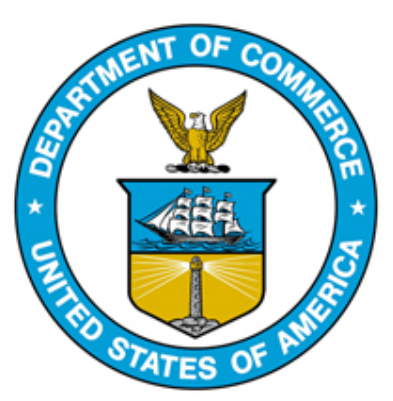

U.S. DEPARTMENT OF COMMERCE

Carlos M. Gutierrez,Secretary TECHNOLOGY ADMINISTRATION

Robert C. Cresanti, Under Secretary of Commerce for Technology NATIONAL INSTITUTE OF STANDARDS AND TECHNOLOGY James M. Turner, Acting Director 


\section{Table of Contents}

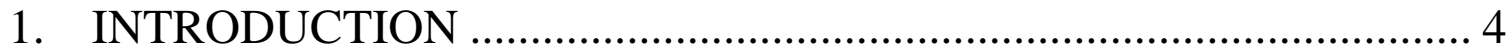

2. QUALITATIVE AND QUANTITATIVE FLUORESCENCE

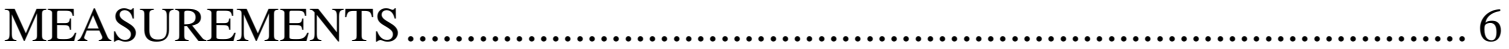

2.1 Qualitative Fluorescence Measurements .......................................................... 6

2.2 Quantitative Fluorescence Measurements ........................................................... 7

3. FACTORS AFFECTING QUANTIFICATION .................................. 7

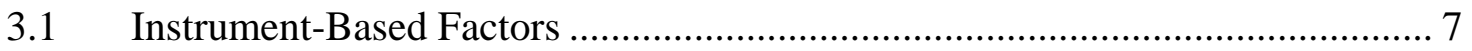

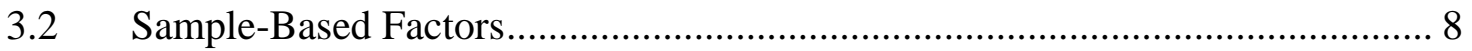

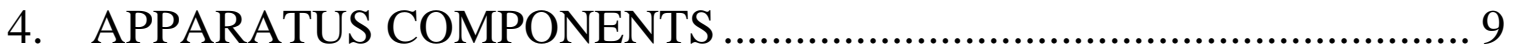

$4.1 \quad$ Excitation Light Source ............................................................................... 10

$4.2 \quad$ Excitation Wavelength Selector....................................................................... 10

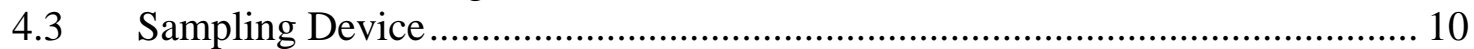

$4.4 \quad$ Emission Wavelength Selector ……………........................................................ 10

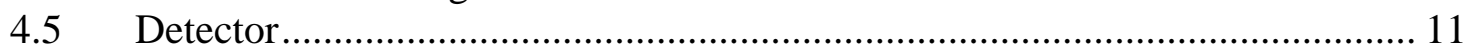

5. CALIBRATION OF FLUORESCENCE INSTRUMENTS ................. 11

5.1 Analyte Concentration - Calibration Curves................................................... 11

5.2 Emission Wavelength and Spectral Slit Width .............................................. 12

5.3 Excitation Wavelength and Spectral Slit Width ................................................ 12

$5.4 \quad$ Linearity of the Detection System .............................................................. 13

5.5 Signal Level (Relative Emission) ………….................................................. 13

5.6 Reference Signal Level (Relative Excitation)................................................ 15

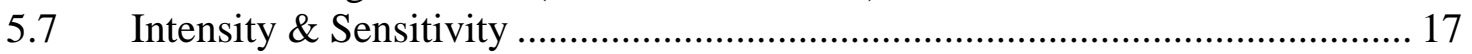

6. QUALIFICATION AND VERIFICATION OF FLUORESCENCE

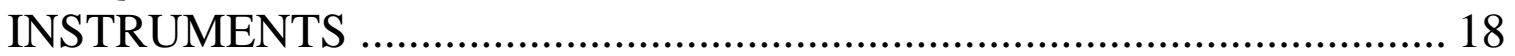

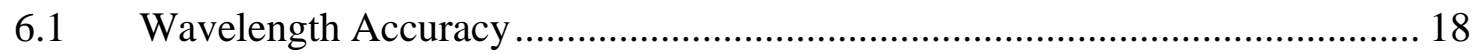

6.2 Signal Level (Relative Emission) ………….............................................. 19

6.3 Reference Signal Level (Relative Excitation).................................................. 19

6.4 Intensity, Sensitivity \& Performance Verification.......................................... 19

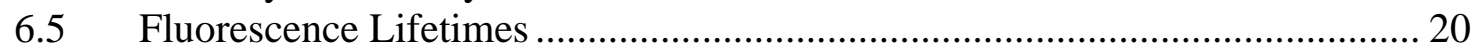

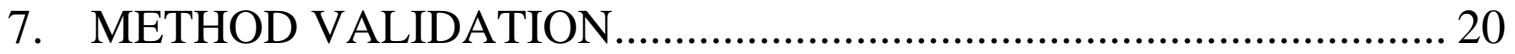

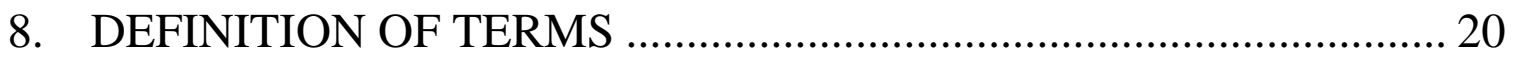

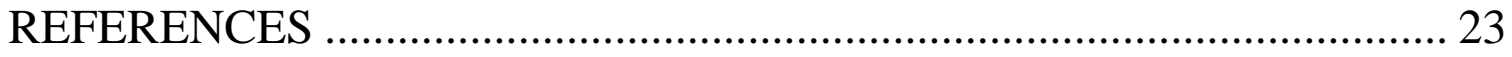




\section{INTRODUCTION}

Fluorescence is a two-step process requiring an initial absorption of light, followed by emission. Fluorescence spectroscopy is an electronic spectroscopic technique related to ultraviolet-visible-near infrared (UV-VIS-NIR) absorbance spectroscopy, but it is also a background-free technique with light emitted from the sample in all directions, similar to Raman spectroscopy. The initial absorption of a photon by a molecule in the sample promotes an electron to an excited state. The excited electron returns to the ground electronic state by emitting a photon. If the emission arises from an "allowed" transition, typically having a short lifetime between 1 ns and 10 ns, then it is called fluorescence. If the emission arises from a "forbidden" transition, typically having a long lifetime between $1 \mathrm{~ms}$ and $1 \mathrm{~s}$, then it is called phosphorescence. Phosphorescence is usually less intense than fluorescence under similar conditions. Fluorescence spectroscopy is specifically discussed in what follows, but many of these issues also apply to phosphorescence. The basic concepts behind fluorescence spectroscopy have been well established [1, 2], but its applications [3, 4] and standardization are still expanding and progressing, making it a developing, not yet mature, technique.

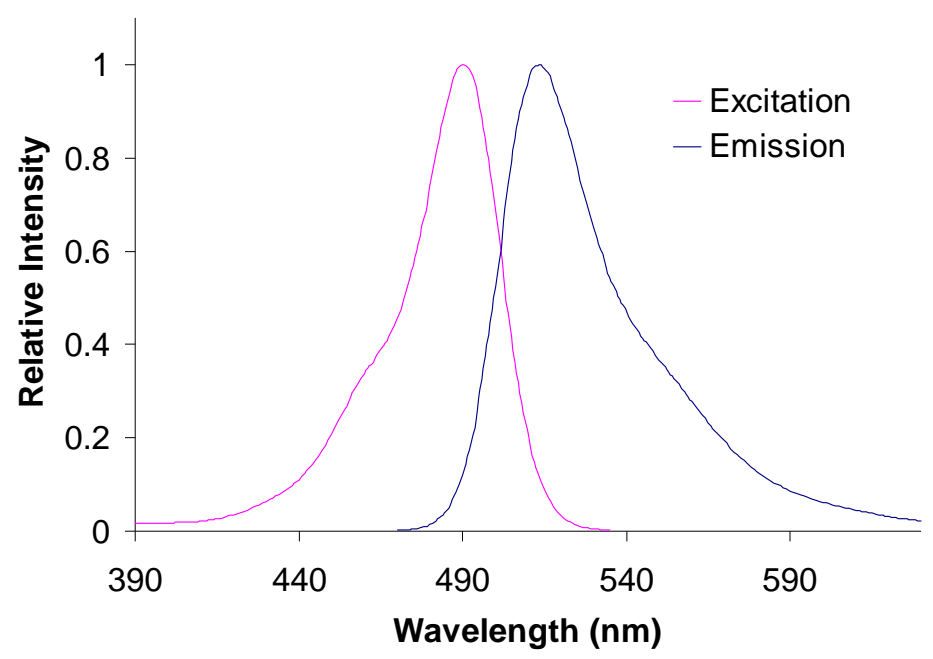

Fig. 1: Fluorescence excitation and emission spectra for fluorescein in borate buffer with the wavelength axis being excitation and emission wavelength, respectively.

The most common type of fluorescent sample is a dilute, transparent solution that absorbs light following the Beer-Lambert law and emits a corresponding fluorescence intensity directly proportional to the concentration, the absorption coefficient and the fluorescence quantum yield of the fluorescent species or fluorophore. A conventional fluorescence spectrometer has both excitation and emission wavelength selectors and a spectrum is collected by fixing the wavelength of one of the selectors and scanning the other wavelength selector over a range. When the excitation wavelength is fixed and the emission wavelength is scanned, the resulting spectrum is termed an emission spectrum. When the emission wavelength is fixed and the excitation wavelength is scanned, the 
resulting spectrum is termed an excitation spectrum. (see Fig. 1) The fluorescence spectrum is plotted as relative intensity or counted photons of fluorescence versus wavelength. The appearance of a fluorescence spectrum is much like a UV-VIS-NIR absorbance spectrum. In fact, the shape or contour of an excitation spectrum is often identical to that of the corresponding absorbance spectrum for an organic dye in solution over the same wavelength range.

Polyatomic fluorophores in condensed media, e.g., solutions, thin films and solids, at room temperature exist in the ground or excited electronic state in a broad distribution of vibrational energy levels, which causes homogeneous broadening of excitation or emission spectra, respectively. A microenvironment or shell also surrounds each fluorophore in condensed media and differences in the structure of this shell between individual fluorophores causes inhomogeneous broadening. These two types of broadening cause fluorescence spectra to be broader than some other types of spectra (e.g., IR absorbance or Raman), with the typical width of a fluorescence band being between $10 \mathrm{~nm}$ and $100 \mathrm{~nm}$. Once electronically excited, a polyatomic fluorophore experiences vibrational relaxation before emitting a photon, causing a red shift, or Stokes shift, of the fluorescence spectrum relative to the wavelength at which it was excited.

Few naturally-occurring biological compounds fluoresce strongly. This apparent disadvantage has been turned into an advantage by the synthesis of fluorescent probes designed to bind to target analytes only. A large number of analyte-specific fluorophores are now commercially available, which bind to a comparable number of biological target molecules. [5, 6] Many of these fluorescent probes, such as fluorescein, rhodamine and their derivatives, have relatively large absorption coefficients and quantum yields close to one, i.e., they fluoresce nearly as many photons as they absorb. Fluorescence techniques are also termed "background-free," since very little excitation light reaches the detector. These advantages make fluorescence detection highly sensitive, down to single molecule detection in some cases. Specificity and sensitivity are two of the most significant strengths of fluorescence techniques. Fluorescence spectroscopy is also typically not destructive to the sample and measurements can be made quickly, on the order of seconds to minutes.

a)

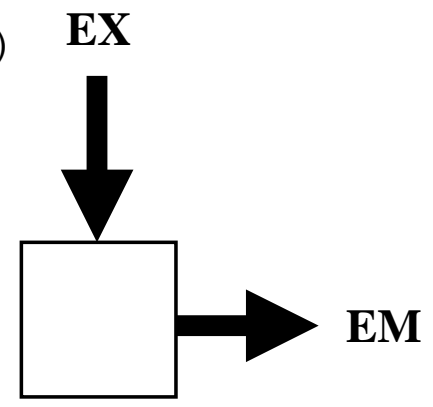

b)

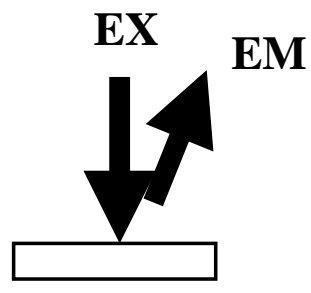

EX

c)

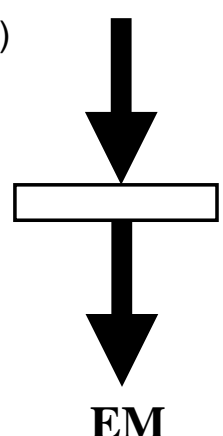

Fig. 2: Schematic of the excitation beam (EX) and detected emission (EM) orientations for a) $0^{\circ} / 90^{\circ}$ right angle transmitting, b) front face and c) $0^{\circ} / 180^{\circ}$ transmitting geometries. 
A right angle or $0^{\circ} / 90^{\circ}$ geometry is typically used to measure dilute solutions and other transparent samples, where the excitation beam is normal to the sample and fluorescence is detected at a $90^{\circ}$ angle relative to the beam (see Fig. 2a). A front-face geometry is used to measure optically dense samples, where the excitation beam is incident on the sample at less than $90^{\circ}$ and the fluorescence is collected at an angle $\leq 90^{\circ}$ (see Fig. 2b). The epifluorescence geometry is a special case of the front-face geometry that is often used in fluorescence microscopy and optical fiber-based fluorometers, where the excitation beam and collected fluorescence are both normal to and on the same side of the sample, i.e., a $0^{\circ} / 0^{\circ}$ geometry. A $0^{\circ} / 180^{\circ}$ transmitting geometry is also often used in microscopy (see Fig. 2c).

The number of chemical assays and screening techniques using fluorescence detection continues to increase rapidly and has resulted in a corresponding increase in the need for standardization of fluorescence measurements. Only a few standard methods [7-9] and reference materials [10-14] have been well established and are readily available, at present, for the characterization of fluorescence measuring systems. National metrology institutes and international standards organizations are working to provide new fluorescence standard materials and methods in the near future. This document briefly discusses the major issues that should be considered by users of fluorescence instruments who aim to achieve high quality measurements. Standard methods and materials are also described where appropriate and references are given where more detailed explanations can be found. A few guideline/recommendation documents have appeared recently [1517], but this one aims to be most useful to non-expert users of fluorescence spectrometers in the bioanalytical, pharmaceutical and clinical communities.

\section{QUALITATIVE AND QUANTITATIVE FLUORESCENCE MEASUREMENTS}

There are two general classes of measurements that are commonly performed by fluorescence spectrometry: qualitative and quantitative.

\subsection{Qualitative Fluorescence Measurements}

Qualitative fluorescence measurements are used to detect the presence of particular analytes, yielding a positive or negative answer. The excitation and emission wavelengths are often fixed at the peak maximum of the fluorophore to be detected. The observation of a fluorescence intensity at the peak position that is greater than a set threshold, typically based on the noise level, e.g., the limit of detection, indicates a positive result.

For more complex systems, e.g., where multiple fluorophores are present, the shape of the fluorescence emission spectrum or the value of the fluorescence lifetime can be used to determine which of the fluorophores of interest are present. 


\subsection{Quantitative Fluorescence Measurements}

Quantitative fluorescence measurements are used to determine amounts or concentrations of analytes in unknown samples. These quantities may be determined in absolute units, such as moles or moles per liter, or in relative units, such as the ratio of the concentrations of two fluorescent analytes contained in a single unknown solution. These determinations use the following proportionality relating fluorescence signal $(S)$ at a given pair of excitation and emission wavelengths $\left(\lambda_{e x}, \lambda_{e m}\right)$ to fluorescent analyte concentration $(c)$ :

$$
\mathrm{S} \propto \mathrm{I}_{0} \Omega \mathrm{R}_{\mathrm{d}} \alpha \Phi \mathrm{C}
$$

where $I_{0}$ is the intensity of the excitation beam, $\Omega$ is the fraction of the fluorescence collected by the detection system, $R_{d}$ is the responsivity of the detection system, and $\alpha$, $\Phi$, and $c$ are the absorption coefficient, fluorescence quantum yield and concentration of the fluorescent analyte, respectively. This linear proportionality with concentration applies to optically dilute samples, e.g., solutions with an absorbance of less than 0.05 at a pathlength of $1 \mathrm{~cm}$.

\section{FACTORS AFFECTING QUANTIFICATION}

\subsection{Instrument-Based Factors [18-23]}

Measurements on a fluorescence instrument require that instrument parameters, such as wavelengths, bandwidths and detector gain, be set. All of these parameters can be set with varying degrees of repeatability and accuracy, depending on the instrument used. This can introduce a measurement uncertainty or bias that is particularly significant when measured values are compared between instruments. For instance, the measured peak positions of the emission bands of two analytes may differ between instruments due to a wavelength bias. A corresponding bias between instruments could be introduced in the results of an assay that depends on the ratio of the fluorescence intensities at the two specified emission wavelengths.

The intensity of the excitation beam can change significantly with excitation wavelength or with time, due to the wavelength-dependence of the intensity of the light source and the transmittance of the excitation wavelength selector or the time-dependence of the

light source intensity, respectively. It is therefore advisable to monitor the excitation beam intensity and correct the measured fluorescence intensity for these fluctuations. This can be particularly important when excitation spectra are being collected, because the excitation intensity often has sharp peaks and valleys with wavelength when lamp sources, such as a Xe lamp, are being used.

The responsivity of a detection system is not linear with intensity at all intensities, making it important to know the linear intensity range of the detection system being used. 
The linear range for most detection systems is from its limit of detection up to a threshold intensity, above which the responsivity becomes increasingly non-linear with increasing intensity. The linear range of the fluorescence detection system should be established before attempting to calibrate the responsivity of the detection system.

The responsivity of the detection system is also wavelength dependent, due to the wavelength dependence of both the transmittance of the emission wavelength selector and the responsivity of the detector. These factors can affect the shape of a measured emission spectrum.

The diffraction efficiency of gratings and the responsivity of detectors is often polarization dependent. This can result in changes in the excitation intensity and the responsivity of the detection system as a result of changing excitation and emission polarization settings. Even when polarizers are not used, the excitation beam may be polarized and the responsivity of the detection system may be polarization dependent and both of these factors will be different for different instruments. Not only can this cause intensity differences, but spectral correction factors will likely change with emission polarization as well.

The passing of multiple wavelengths by a diffraction grating can introduce unexpected sharp peaks into a fluorescence spectrum. A particular wavelength is chosen using a diffraction grating by setting the angle of the grating with respect to the incident light, such that the incident light is diffracted at the desired wavelength according to the grating equation. The value of $m \lambda$, not $\lambda$, is fixed according to this equation, where $m$ is an integer, termed the "diffraction order." Therefore, the grating equation can be satisfied by more than one wavelength for a single grating position. For instance, if a grating in an emission monochromator is set to pass $500 \mathrm{~nm}$ light at first order, it will also pass $250 \mathrm{~nm}$ light at second order. As a result, the scattered light from a $250 \mathrm{~nm}$ excitation beam will be detected as a peak at an emission wavelength of $500 \mathrm{~nm}$.

\subsection{Sample-Based Factors [24-26]}

The fluorescence intensity of optically dense samples (e.g., A $>0.05$ at a pathlength of 1 $\mathrm{cm}$ ) does not increase linearly with concentration due to significant absorption of the excitation beam and/or the emission (reabsorption) by the sample. These "inner filter effects" can also greatly reduce the amount of fluorescence that reaches the detector, especially for a right angle transmitting geometry. The fluorescence intensity can also become strongly dependent on sample position and optical geometry. At even higher concentrations (e.g., A>>0.05 at a pathlength of $1 \mathrm{~cm}$ ) aggregation of fluorophores often occurs, causing the shape of the fluorescence spectrum to be different than that of a dilute sample, along with non-linear concentration behavior.

The fluorescence intensity of a sample may decrease with exposure time to light, due to photobleaching and photodegradation. This is particularly true of most organic dyes, which are the most widely used fluorescent probes. The light exposure time of such 
samples may need to be limited to obtain reproducible fluorescence intensities and in some cases even reproducible spectral shapes.

The fluorescence intensity of fluorophores is temperature dependent. Typically, the rates of fluorescence quenching processes, such as collisional quenching in solutions, increase with temperature, causing a corresponding decrease in fluorescence intensity. Temperature coefficients for fluorescence intensity for particular fluorophores can be used to correct for this temperature dependence.

The absorbance and consequently the intensity of fluorescence from a sample are dependent on the orientation of the sample's absorption transition dipole with respect to that of the polarization of the excitation light. The polarization of fluorescence is parallel to the direction of polarization of the fluorescent species' emission transition dipole. Fluorescence anisotropy ( $\mathrm{r}$ ) is used to describe the extent of polarization of emission and is defined by $r=\left(I_{l l}-I_{\perp}\right) /\left(I_{l l}+2 I_{\perp}\right)$, where $I_{l l}$ and $I_{\perp}$ are the observed fluorescence intensities when the fluorometer's emission polarizer is oriented parallel and perpendicular, respectively, to the direction of the polarized excitation. A sample whose fluorophores are oriented non-randomly and have a rotational period that is long compared to their fluorescence lifetime will emit anisotropic fluorescence. The spectral shape and intensity of such fluorescence is dependent on the viewing angle and the polarization factors of the instrument.

It is common for the fluorescence intensity and peak position, and sometimes even the spectral shape, of a fluorophore to be environment-dependent. This includes changes due to the solvent used, the $\mathrm{pH}$ of a solution or the species to which a fluorophore is bound. This can be of particular concern when reference samples containing the same fluorophores as an unknown sample, but in a different environment, are used to quantify the amount of analyte in the unknown.

Raman signal can introduce peaks into the fluorescence spectrum. The Raman peaks of the sample's solvent or matrix being those most commonly encountered. For instance, the Raman peak of water, found red-shifted about $3400 \mathrm{~cm}^{-1}$ from the chosen excitation wavelength, would typically be observed in the fluorescence spectrum of any aqueous solution excited by UV or blue light.

\section{APPARATUS COMPONENTS}

All modern fluorescence measurements involve irradiating a sample with a light source, selecting the excitation wavelength, collecting the resulting fluorescence, rejecting the Rayleigh-scattered light, selecting the emission wavelength and detecting the fluorescence signal. The following functions will be discussed individually along with the equipment used to achieve these functions in commercial instruments:

1. Excitation Light Source

2. Excitation Wavelength Selector 
3. Sampling Device

4. Emission Wavelength Selector

5. Detector

\subsection{Excitation Light Source}

A variety of lamps, lasers and light-emitting diodes (LEDs) are used as an excitation source. Continuous and pulsed versions of these sources are used for steady-state and time-resolved instruments, respectively. The Xe lamp is the most commonly used of these, due to its relatively high intensity and broad wavelength range (UV to NIR). Lasers are the highest intensity sources and are used in applications where short collection times and small amounts of sample are required, such as those using flow cytometry or microarrays.

\subsection{Excitation Wavelength Selector}

The intensity of scattered light at the excitation wavelength can be comparable to or greater than that of the fluorescence at the sample. Therefore, it is important that the excitation wavelength profile does not overlap with the emission wavelength region being detected. This is achieved for lamps by using an excitation wavelength selector (e.g., a filter or a monochromator) between the lamp and sample with a known peak transmission wavelength and bandwidth. The inherent bandwidth of the radiation from a laser or an LED is often narrow enough that an excitation wavelength selector is not necessary. This selector also enables fluorescence excitation spectra to be resolved.

\subsection{Sampling Device}

The sampling device includes all optics and other equipment needed to deliver the excitation beam to the sample, collect the emission from the sample and hold the sample in place. Sample formats include cuvettes, microwell plates, microarrays, microscope slides and flow systems and can be accompanied by a variety of optical delivery and collection systems, including conventional transmitting, front face and epifluorescence systems and fiber optic-based probes.

\subsection{Emission Wavelength Selector}

Similar to that for excitation, the emission wavelength selector helps to insure that the emission wavelength region being detected does not overlap with the excitation wavelength profile. In addition, it enables individual fluorescence bands to be detected when multiple bands are present and fluorescence emission spectra to be resolved. Emission wavelength selectors are also important for the rejection of stray light. Filters, 
monochromators and grating polychromators are most often used for emission wavelength selection.

\subsection{Detector}

For the detection of emission, a photomultiplier tube (PMT) or a CCD array is typically placed after the emission wavelength selector. The detection of the excitation beam for monitoring its intensity is commonly done by a quantum counter detector or a photodiode before the sample, where a small fraction of the excitation beam is split off from the rest.

\section{CALIBRATION OF FLUORESCENCE INSTRUMENTS [15, 16, 18- 23]}

Two types of fluorescence instrument calibrations are used. The first is analyte-specific, where the relationship between the response of the instrument (fluorescence intensity) and the concentration or amount of a specific analyte is determined for the sample of interest. This is the most commonly used type of calibration for fluorescence. The second is analyte-independent and intended for spectral instruments. In this case, the wavelength accuracy for emission and/or excitation and the spectral responsivity of the detection system are calibrated across the entire or a continuous part of the wavelength range of the instrument.

\subsection{Analyte Concentration - Calibration Curves}

Calibration curves of instrument response (fluorescence intensity) versus analyte concentration are determined using reference materials containing the analyte of interest. For instance, the fluorescence intensities of a set of solutions at different, known analyte concentrations, which covers a desirable concentration range, can be measured and plotted versus concentration. The plot is then fitted to a polynomial, typically a straight line. The resulting calibration curve is both analyte and instrument specific and can be used to determine analyte concentrations of unknown samples. [8, 14] Note that this method may not be accurate when the microenvironment surrounding the fluorophore is different in the reference and unknown samples. In addition, users must insure that the fluorescence intensities of samples are reproducible and do not decrease over the time period that they are being excited and measured, because the organic dyes typically used can be prone to photobleaching.

It is not always possible to use this method effectively. Firstly, organic dyes, which are used as fluorescent probes, are often not commercially available at a known, high purity to enable reference solutions to be produced. Secondly, in complex systems where fluorophores are bound to large molecules, cells or microbeads, the concentration of bound fluorophores in a solution or suspension may be very difficult to determine. For the latter case, the molecules of equivalent soluble fluorophore (MESF) scale [27-29] has 
been proposed as an alternative way to use calibration curves to quantify fluorescence intensity for a particular analyte.

\subsection{Emission Wavelength and Spectral Slit Width}

A variety of reference samples have been proposed for use in the determination of emission wavelength accuracy, including several lamps, and inorganic and organic fluorophores. The most widely used and best characterized of these are low pressure atomic lamps, commonly referred to as "pen" lamps. In this case, the type of pen lamp (e.g., $\mathrm{Hg}, \mathrm{Ne}, \mathrm{Kr}$, etc.) is chosen, so that its radiated atomic lines are within the desired wavelength range. The lamp is placed at the sample position, such that its light is centered in the optical path of the detection system of the instrument. Note that the accuracy of this method may decrease if the pen lamp is not properly aligned. The emission wavelength selector with detector then measures the signal over the wavelength range of interest. The measured wavelength positions of the resulting sharp peaks are then compared with the known positions to determine wavelength accuracy. $[7,16]$

Spectral slit width accuracy of the emission wavelength selector can be determined by measuring the spectral bandwidth, taken to be the full width at half the peak maximum (FWHM), of a single line of a pen lamp. [7] For fluorescence spectrometers with both excitation and emission monochromators, an alternative method may be used where one monochromator is scanned over the position of the other. [18]

\subsection{Excitation Wavelength and Spectral Slit Width}

Many of the reference samples that can be used for determining emission wavelength accuracy can also be used for excitation wavelength accuracy. For instance, a pen lamp can be placed at the excitation light source position and detected after the excitation wavelength selector using the instrument's reference detector. [19] In this case, a relatively weak signal that limits the number of useful atomic lines is likely to be observed and alignment of the pen lamp is more critical than for the emission wavelength accuracy determination.

Once the accuracy of the emission wavelength selector has been determined, a diffuse scatterer, e.g., a scattering solution or a diffuse reflector, at the sample can be used to scatter a fraction of the excitation beam into the detection system to determine excitation wavelength accuracy. One wavelength selector is set at a fixed wavelength while the other is tuned over the same wavelength to obtain a spectrum. The wavelength bias between the two wavelength selectors is equal to the difference between the set wavelength position and the observed peak position of the collected spectrum. This method $[7,18]$ can be used at any wavelength, unlike many other methods that depend on a limited number of set excitation wavelengths determined by the reference material chosen. Methods similar to those used for spectral slit width accuracy of the emission 
wavelength selector may also be used for spectral slit width accuracy of the excitation wavelength selector. $[7,18]$

\subsection{Linearity of the Detection System}

Several methods can be used to determine the linear intensity range of the detection system. They can be separated into three types, based on the tools used to vary the intensity of light reaching the detector: 1) double aperture, 2) optical filters and/or polarizers, 3) fluorophore concentrations. The double aperture method is the most well established and probably the most accurate when done correctly, but is also the most difficult to perform. [30, 31] A variety of methods using optical filters, polarizers or a combination of the two have been reported. $[18,19]$ These methods require high quality, often costly, components and some expertise on the part of the user. The third method is the most popular and easiest to implement. It uses a set of solutions obtained by serial dilution of a fluorescent stock solution, similar to that used for obtaining calibration curves for analyte concentration, as described in section 5.1. In this case, solutions with low concentration ( $\mathrm{A}<0.05$ at $1 \mathrm{~cm}$ pathlength) should be used and fluorophore adsorption to cuvette walls may affect measurements at vey low concentrations. [8] Users must insure that the fluorescence intensities of samples are reproducible and do not decrease over the time period that they are being excited and measured, because the organic dyes typically used can be prone to photobleaching.

\subsection{Signal Level (Relative Emission) [21, 23 , 18-20]}

Calibration of the relative responsivity of the emission detection system with emission wavelength, also referred to as spectral correction of emission, is necessary for successful quantification when intensity ratios at different emission wavelengths are being compared or when the true shape or peak maximum position of an emission spectrum needs to be known. Such a calibration is necessary because the relative spectral responsivity of a detection system can change significantly over its wavelength range (see Fig. 3). The degree of photometric precision required for the successful outcome of quantitative methods should be known. It is highly recommended that the wavelength accuracy (see section 5.2) and linear range of the detection system be determined (see section 5.4) before this calibration is performed and that appropriate steps are taken (e.g., the use of attenuators) to insure that all measured intensities during this calibration are within the linear range. Also note that when using an emission polarizer, the spectral correction for emission is dependent on the polarizer setting. In addition, users must be careful to insure that signal intensities and correction factors are expressed consistently, either in power units or in photon units. Some detectors, such as those with photon-counting capabilities and quantum counter detectors, give a signal that is proportional to the number of incident photons, while other detectors give a signal that is proportional to the incident power. Energy and photon signals can be interconverted easily (see Planck's constant in section 8). 
There are two preferred methods for calibrating photometric responsivity. One (method A) uses a calibrated light source (CS) and the other (method B) uses certified reference materials (CRMs). Both give results that are traceable to national metrology institutes (NMIs). A calibrated tungsten white light source is most commonly used for method A, covering the wavelength range from about $350 \mathrm{~nm}$ into the NIR. NIST SRMs [10-12] and BAM CRMs [13] are currently available for use in method B. Corrected emission spectra of some commonly used dyes have also been reported recently in the literature. [32, 33] Method A is more difficult to implement than method $\mathrm{B}$ and requires periodic recertification of the CS, which is more expensive over time. A third method, method C, can be used, implementing a calibrated detector and a calibrated diffuse reflector. This method typically has larger uncertainties associated with it than methods A and B, [18] but is recommended in wavelength regions in the UV and NIR not covered by the other two methods.

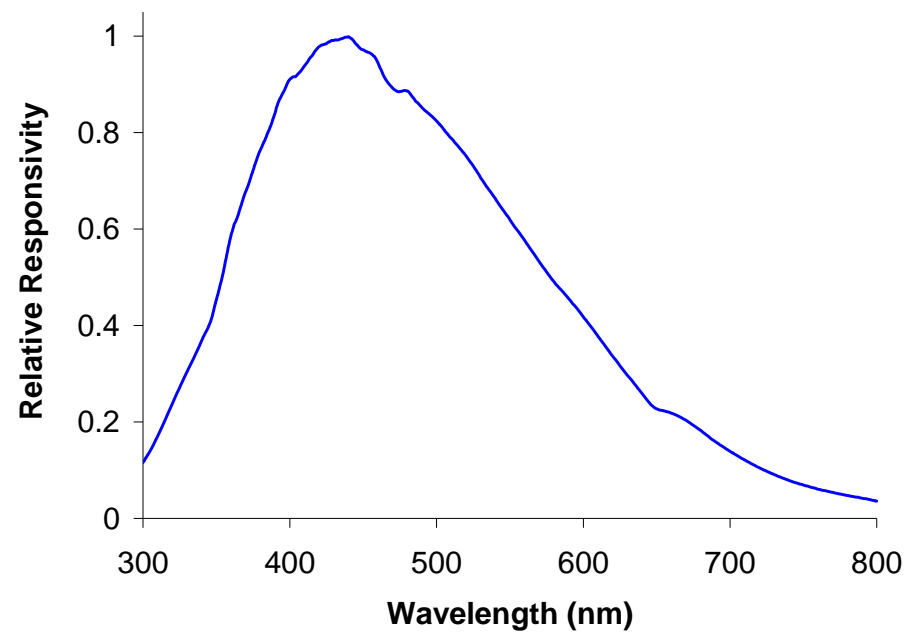

Fig. 3: Example of the relative spectral responsivity of an emission detection system (grating monochromator-PMT based), [18] for which a correction needs to be applied to a measured emission spectrum to obtain its true spectral shape (relative intensities).

Method A - The light from a calibrated source (CS) is directed into the emission detection system by placing the CS at the sample position. If the CS is too large to be placed at the sample position, a calibrated diffuse reflector (CR) may be placed at the sample position to reflect the light from the CS into the emission detection system. The emission wavelength selector is scanned over the emission region of interest, using the same instrument settings as that used with the sample, and the signal channel output ( $\left.\mathrm{S}^{\prime \prime}\right)$ is collected. The known radiance of the CS incident on the detection system (L) can be used to calculate the relative correction factor $\left(\mathrm{C}_{\mathrm{CS}}\right)$, such that $\mathrm{C}_{\mathrm{CS}}=\mathrm{L} / \mathrm{S}^{\prime \prime}$. The corrected emission intensity is equal to the product of the signal output of the sample (S) and $\mathrm{C}_{\mathrm{CS}}$.

Method B - The fluorescence standard is placed at the sample position. Its spectrum is collected and compared to the certified spectrum according to the instructions given on the accompanying certificate, yielding spectral correction factors for the instrument. 
Method C - This is a two-step method. The first step uses a calibrated detector (CD) at the sample position to measure the flux of the excitation beam as a function of excitation wavelength. The second step uses a calibrated diffuse reflector (CR) to reflect a known fraction of the flux of the excitation beam into the detection system. This is done by placing the $\mathrm{CD}$ at the sample position at a $45^{\circ}$ angle, assuming a $0^{\circ} / 90^{\circ}$ instrument geometry, and synchronously scanning both the excitation and emission wavelength selectors over the emission region of interest while collecting both the signal output and the reference output. This method enables the relative correction factor to be calculated. Note that this method has larger uncertainties than those for methods A or B and is typically more difficult to implement.

\subsection{Reference Signal Level (Relative Excitation) [22, 23 , 18-20]}

Calibration of the excitation intensity with excitation wavelength is necessary for successful quantification when intensity ratios at different excitation wavelengths are being compared or when the true shape or peak maximum position of an excitation spectrum needs to be known. Such a calibration is necessary because the relative spectral flux of an excitation beam at the sample can change significantly over its wavelength range (see Fig. 4). The neglect of excitation intensity correction factors can cause even greater errors than that of emission correction factors. [18, 34] Fortunately, many fluorescence instruments have a built-in reference detection system to monitor the intensity of the excitation beam. This is usually done using a photodiode or a PMT, or a quantum counter detector to measure a fraction of the excitation beam that is split off from the rest of the beam. The collected reference signal can be used to correct the fluorescence signal for fluctuations due to changes in the excitation beam intensity. Reference detectors are often not calibrated with excitation wavelength, introducing errors, which can be particularly large over longer excitation wavelength ranges (e.g., greater than $50 \mathrm{~nm}$ ) or in a wavelength region where the excitation intensity changes rapidly with excitation wavelength, such as the UV. It is highly recommended that the wavelength accuracy of the excitation wavelength selector (see section 5.3) and linear range of the detection system (see section 5.4) used to measure the excitation beam be determined before a spectral correction of the excitation beam is performed and that appropriate steps are taken (e.g., the use of attenuators) to insure that all measured intensities during this calibration are within the linear range. Signals and correction factors must be expressed consistently in either power or photon units (see section 5.5). Also note that when using an excitation polarizer, the spectral correction for excitation intensity is dependent on the polarizer setting. 


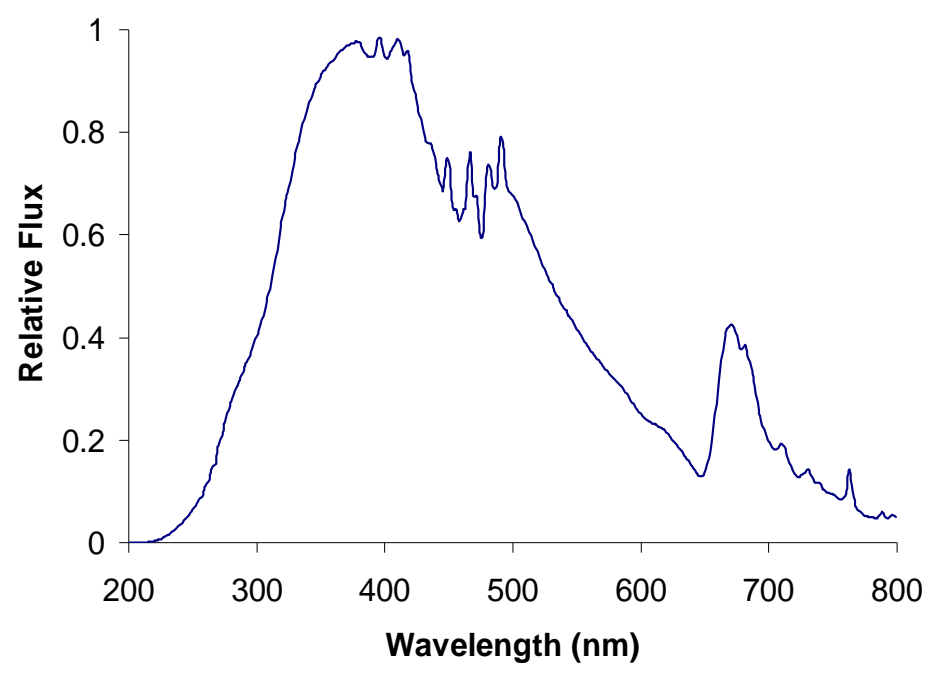

Fig. 4: Example of the relative flux of an excitation beam (Xe lampgrating monochromator based), [18] for which a correction needs to be applied to a measured excitation spectrum to obtain its true spectral shape (relative intensities).

When a reference detector is not built into an instrument, a spectral correction for the reference channel is to be determined, or an independent spectral correction of excitation intensity is desired, 1) a calibrated detector, 2) a calibrated diffuse reflector or 3) a quantum counter [20,35,36] may be placed at the sample position, with the latter two methods using the instrument's fluorescence detection system as a detector. For methods 1 and 2, the detector and diffuse reflector must be calibrated for responsivity and reflectance, respectively, as a function of wavelength. For method 2, excitation and emission wavelength selectors must be scanned synchronously and the spectral correction for the emission channel (see section 5.5) must be applied to the measured intensities. [18] Method 3 should only be used in the quantum counter's effective wavelength range, where a wavelength-independent response can be achieved. This method is probably the least expensive of the three to implement, as no calibrations and re-calibrations are required. Method 1, using a calibrated detector (CD), has fewer caveats associated with it than the other two methods.

Method 1 - A CD is put at the sample position with the excitation beam incident on it. The output of the CD is measured as a function of emission wavelength by scanning the excitation wavelength selector over the excitation region of interest using the same instrument settings as that used with the sample. The known responsivity of the CD is used to calculate the flux of the excitation beam. If the instrument's reference detector is used to measure the intensity of the excitation beam simultaneously with the CD, then the correction factor for the responsivity of the reference detector can also be calculated. 


\subsection{Intensity \& Sensitivity}

As explained in the Quantitative Fluorescence Measurements section, the absolute value of the fluorescence signal measured by the detection system is not only dependent on the sample itself, but also on the excitation intensity at the sample and the optical geometry of the instrument. Therefore, it is non-trivial to determine the instrument-independent fluorescence intensity of any sample or the absolute responsivity of any detection system in terms of the intensity coming out of the sample or being measured by the detector, respectively, relative to the excitation intensity at the sample.

The most accurate way to calibrate an instrument for absolute intensity is to use conventional physical transfer standard-based methods, such as those employing a calibrated light source or a calibrated detector in combination with a calibrated reflector. $[18,19]$ These methods are difficult to perform and require a certain degree of user skill and knowledge. The certification and recertification, typically annual, of such standards is also expensive. In addition, these standards tend to be bulky and are not compatible with many instruments. More simple, easy-to-use, alternative standards and methods are, therefore, used by most researchers.

One approach is to relate fluorescence signal to analyte concentration, using calibration curves or MESF units (see section 5.1). Another approach is to measure the intensity of a standard sample that can be expected to always give the same fluorescence intensity under the same conditions.

Organic dyes, such as those used as fluorescent probes, are not generally good choices for intensity standards, due to issues with photobleaching, stability and reproducible concentration. If organic dyes are to be used in this way, then those with known high purity and known shelf-life, such as those produced by NMIs are recommended for single-time use, i.e., a fresh solution should be used every time.

A better alternative is to use fluorescent samples that are stable over time even when exposed to light. For example, fluorescent, inorganic glasses with well characterized photostability and spectral properties and long shelf-lives are commercially available. $[10,11,24]$ Such materials can be used for determining a quasi-absolute intensity scale by measuring fluorescent signal at fixed wavelength values within their recommended range, using a specified set of experimental parameters, such as bandwidths, excitation intensity and temperature.

The sensitivity of a fluorescence instrument can also be determined by measuring the signal-to-noise ratio of the fluorescence signal of intensity standards. The Raman line of water is often used to measure sensitivity in a similar way, [37-39] but the Raman signal is typically only strong enough to be useful in the UV region. Organic dye solutions can also be used to measure instrument sensitivity or limit of detection with all of the same caveats as when used as intensity standards. [9] 
The methods outlined above enable a quasi-absolute intensity scale to be defined that is expected to be instrument independent for instruments with similar optical geometries and designs, potentially enabling the sensitivity of different fluorescence instruments to be compared, but such comparisons should be approached with caution due to the relatively large uncertainties involved, which are difficult to quantify. [18]

\section{QUALIFICATION AND VERIFICATION OF FLUORESCENCE INSTRUMENTS}

Instrument qualification is an essential part of method validation, when the results of the method are dependent on the performance of the instrument. Instrument qualification should be done at well-defined, periodic intervals or after significant changes or maintenance on the instrument has been carried out. In between qualifications, more frequent (possibly daily) performance verifications should be done to demonstrate that the instrument is performing at a consistent quality level.

Many of the methods in the Calibration section may be appropriate for qualification and verification of fluorescence instruments. The instrumental variables and desired accuracy of the quantities to be determined will define the particular types of test methods needed. The criteria for acceptable instrument performance given in the following subsections are applicable for general use unless specified otherwise. Two general types of instrumental measurements will be differentiated here, spectral, i.e., those that measure intensity versus wavelength, and fixed, i.e., those that measure intensity at a fixed wavelength and bandwidth.

\subsection{Wavelength Accuracy}

The confidence level of measured peak positions is defined by wavelength accuracy for spectral measurements. Determining and calibrating the wavelength accuracy (see sections 5.2 and 5.3) at a single point (one for emission and one for excitation) is often sufficient to qualify an instrument for this parameter, as it is common for the relative wavelength accuracy to not change dramatically, e.g., on the order of $1 \mathrm{~nm}$, across an instruments effective wavelength range. Determination of the accuracy of many wavelengths across the desired wavelength range demonstrates if further calibration, beyond a single point, is needed. Multi-point calibration involves measuring wavelength biases at multiple wavelengths and correcting for the wavelength dependence of the bias. A wavelength uncertainty of $\pm 0.2 \mathrm{~nm}$ has been demonstrated for a scanning fluorescence spectrometer. [18] A single point calibration can often be applied to the wavelength axis in an instrument's software before data is collected. Whereas, a multi-point calibration may require that the correction be applied to spectra after they are collected.

For fixed measurements, it is most important that the wavelength position and bandwidth be reproducible. For filter-based wavelength selection, this just requires that the same filter be used when comparing data over time. If a different filter has to be used, for 
instance when data is compared across instruments and laboratories, then the transmission curves of the filters must be compared.

\subsection{Signal Level (Relative Emission)}

Correction for the relative responsivity of the detection system with emission wavelength (see section 5.5) is important for comparing spectral shapes and integrated intensities of emission spectra, and intensities at different emission wavelengths. Note that the linearity of the detection system must be determined before a calibration of spectral emission is performed (see section 5.4). Corrected emission spectra are required for obtaining accurate results for assays that depend on such intensity values and for quantum yield determinations. Spectral correction is particularly needed in emission regions where the responsivity of the detection system is highly sloped, typically in the UV and red/NIR. For filter-based wavelength selection, the detected spectral range is determined by the transmission profile of the filter, which changes from one filter to another.

\subsection{Reference Signal Level (Relative Excitation)}

Correction for the relative spectral flux of the excitation beam at the sample (see section 5.6) is critical for accurate determination of fluorescence intensities at different excitation wavelengths and the shape and peak positions of excitation spectra. The spectral profile of a broadband excitation source, such as a high pressure atomic lamp, may, in fact, exhibit even more dramatic spectral changes with wavelength than typical detection systems. This problem is compounded by possible changes of the source intensity with time, even at a fixed wavelength. The relative spectral flux of a source or the relative spectral responsivity of a reference detector (see section 5.6), should be determined periodically, along with correction factors possessing the accuracy and precision required for the application.

\subsection{Intensity, Sensitivity \& Performance Verification}

The use of photostable, day-to-day intensity standards (see section 5.7) is the easiest way to verify the performance of an instrument. If the measured intensity does not change from that observed when the instrument was qualified, then it can be assumed that the instrument performance has not changed and is, therefore, still qualified. Using such standards to determine an artifact-based or quasi-absolute intensity scale potentially enables measured intensities and instrument sensitivity to be compared over time or between instruments. Note that it is important to demonstrate that intensity measurements are being measured within the linear range of the instrument's detection system before intensity comparisons are attempted (see section 5.4).

For instruments with filter-based wavelength selection, fluorescence standards for spectral correction can be used to determine expected intensity differences caused by 
filters with different transmission profiles. By compensating for these intensity differences due to spectral mismatch, a quasi-absolute intensity scale can potentially be determined for these instruments, as well. Again, note that instrument-to-instrument comparisons should be approached with particular caution due to the relatively large uncertainties involved, which are difficult to quantify. [18]

\subsection{Fluorescence Lifetimes}

Time-domain and frequency-domain measurements are the two types of fluorescence measurements used to determine fluorescence lifetimes. [40] Conventional instruments include those based on time-correlated single-photon counting (time-domain) and multifrequency phase and modulation (frequency-domain) techniques, which are typically used to measure lifetimes from picoseconds to microseconds. More simple, time-domain instruments are commonly used to measure lifetimes on the order of milliseconds or longer. Criteria for fluorescence lifetime standards include 1) high purity, 2) a single exponential decay component, and 3) a lifetime independent of excitation and emission wavelengths. Possibly, the most thorough comparison of fluorescence lifetime candidates was recently performed by nine expert laboratories. [41] Almost all of the candidates mentioned in the literature for use as lifetime standards [42] have been liquid, organic dye solutions, probably due to the more complex, excited-state kinetics that exist in most solid fluorescent samples. Lifetime standards are measured in the same way as typical unknown samples. A bias in the measured lifetime or an observed multiexponential decay of the standard indicates the presence of systematic errors in the instrument.

\section{METHOD VALIDATION}

Validation of an analytical method demonstrates that the result yielded from the method is valid within a specified, acceptable uncertainty budget. For fluorescence measurements this involves the consideration of one or more of the topics already discussed. Instrument qualification (see section 6), which may also involve instrument calibration (see section 5 ), is usually part of the process. Sample related errors may also need addressing (see section 3.2). These can arise due to the effects of concentration, anisotropy, photostability and shape of the sample in combination with the optical geometry of the instrument on measured quantities, such as fluorescence intensity. All suspected errors are quantified and combined to give a total estimated error that must be less than the method-specific, acceptable limit. [43]

\section{DEFINITION OF TERMS [44, 45]}

absorption coefficient $(\alpha)$ - a measure of absorption of radiation from an incident beam as it traverses a sample according to Bouguer's law, $\mathrm{I} / \mathrm{I}_{0}=\mathrm{e}^{-\alpha \mathrm{b}}$, where $\mathrm{I}$ and $\mathrm{I}_{0}$ are the 
transmitted and incident intensities, respectively, and $\mathrm{b}$ is the pathlength of the beam through the sample. Note that transmittance $\mathrm{T}=\mathrm{I} / \mathrm{I}_{\mathrm{o}}$ and absorbance $\mathrm{A}=-\log \mathrm{T}$

Beer-Lambert law (or Beer's law or Beer-Lambert-Bouquer law) - relates the dependence of the absorbance (A) of a sample on its pathlength (see absorption coefficient, $\alpha$ ) and concentration (c), such that $A=\alpha b c$

calibrated detector (CD) - a light detector whose responsivity as a function of wavelength has been determined along with corresponding uncertainties. [46]

calibrated light source (CS) - a light source whose radiance as a function of wavelength has been determined along with corresponding uncertainties. [47, 48]

calibrated diffuse reflector (CR) - a Lambertian reflector whose reflectance as a function of wavelength has been determined along with corresponding uncertainties. [49]

certified reference material (CRM) - a material with properties of interest, whose values and corresponding uncertainties have been certified by a standardizing group or organization.

diffuse scatterer - a material that scatters light in multiple directions; this includes diffuse reflectors, which are often Lambertian, and scattering solutions, which are not Lambertian.

fluorescence anisotropy (r) - a measure of the degree of polarization of fluorescence, defined as $r=\left(I_{l l}-I_{\perp}\right) /\left(I_{l l}+2 I_{\perp}\right)$, where $I_{l l}$ and $I_{\perp}$ are the observed fluorescence intensities when the fluorometer's emission polarizer is oriented parallel and perpendicular, respectively, to the direction of the polarized excitation.

fluorescence band - a region of a fluorescence spectrum where the intensity passes through a maximum, usually corresponding to a discrete electronic transition

fluorescence lifetime - parameter describing the time decay of the fluorescence intensity of a sample component; if a sample decays by first-order kinetics, this is the time required for its fluorescence intensity and corresponding excited state population to decrease to 1/e of its initial value.

fluorescence quantum efficiency - the ratio of the number of fluorescence photons leaving an emitter versus the number of photons absorbed.

fluorescence quantum yield $(\Phi)$ - the probability that a molecule or species will fluoresce once it has absorbed a photon. This quantity is an innate property of the species and is typically calculated for a sample as the ratio of the number of molecules that fluoresce versus the number of molecules that absorbed. 
flux (or radiant flux) - rate of propagation of radiant energy, typically expressed in watts (W); spectral flux is the flux per unit spectral bandwidth, typically expressed in W / nm.

grating equation - describes the relationship between the angle of diffraction and wavelength of radiation incident on a grating, i.e., $m \lambda=d(\sin \alpha+\sin \beta)$, where $d$ is the groove spacing on the grating, $\alpha$ and $\beta$ are the angles of the incident and diffracted wavefronts, respectively, relative to the grating normal and $\mathrm{m}$ is the diffraction order, which is an integer.[50]

inner filter effects - a decrease in the measured quantum efficiency of a sample due to significant absorption of the excitation beam and /or reabsorption of the emission of the sample by itself. This causes the measured quantum efficiency to be dependent on the absorbance, concentration, and excitation and emission pathlengths of the sample. [51, 52]

intensity - a measure of the amount of electromagnetic energy present. This general definition, which is used here, is synonymous with or directly proportional to the signal output of a photodetector or the flux of a sample or light source. A more specific definition, often used in radiometry, is "the radiant flux per unit solid angle from a point source," which is typically expressed in W/sr.

Lambertian reflector - a surface that reflects light according to Lambert's law, i.e., the light is unpolarized and has a radiance that is isotropic or independent of viewing angle.

limit of detection - an estimate of the lowest concentration of an analyte that can be measured with a given technique, often taken to be the analyte concentration with a measured signal to noise ratio of three.

noise level - the peak-to-peak noise of a blank

photobleaching - a loss of emission or absorption intensity by a sample due to exposure to light. This loss can be reversible or irreversible with the latter typically referred to as photodegradation or photodecomposition.

Planck's constant (h) - relates the energy (E) of a photon to its frequency ( $v)$, such that $\mathrm{E}=\mathrm{h} v$.

quantum counter - a photoluminescent emitter with a quantum efficiency that is independent of excitation wavelength over a defined spectral range. When a quantum counter is combined with a detector to give a response proportional to the number of incident photons, the pair is called a quantum counter detector.

quasi-absolute fluorescence intensity scale - a fluorescence intensity scale that has been normalized to the intensity of a fluorescent reference sample or artifact under a fixed set of instrumental and experimental conditions. This artifact should be known to 
yield a fluorescence intensity that is reproducible with time and between instruments under the fixed set of conditions.

Raman scattering - inelastic scattering of radiation (the wavelengths of the scattered and incident radiation are not equal) by a sample that occurs because of changes in the polarizability of the relevant bonds of a sample during a molecular vibration. The radiation being scattered does not have to be in resonance with electronic transitions in the sample, unlike fluorescence. [53]

Rayleigh scattering - elastic scattering of radiation by a sample, i.e., the scattered radiation has the same energy (same wavelength) as the incident radiation.

responsivity (spectral) - ratio of the photocurrent output and the radiant power collected by a light detection system. Spectral responsivity is the responsivity per unit spectral bandwidth.

sensitivity - a measure of an instrument's ability to detect an analyte under a particular set of conditions

spectral bandwidth (or spectral bandpass or resolution) - a measure of the capability of a spectrometer to separate radiation or resolve spectral peaks of similar wavelengths.

spectral slit width - the mechanical width of the exit slit of a spectrometer divided by the linear dispersion in the exit slit plane.

transition dipole moment - an oscillating dipole moment induced in a molecular species by an electromagnetic wave that is resonant with an energy transition of the species, e.g., an electronic transition. Its direction defines that of the transition polarization, and its square determines the intensity of the transition.

\section{REFERENCES}

1. Lakowicz, J.R., "Principles of Fluorescence Spectroscopy," second edition, Kluwer Academic/Plenum Publishers, New York (1999).

2. Valeur, B., "Molecular Fluorescence, Principles and Applications," Wiley-VCH, Weinheim (2002).

3. Lakowicz, J.R., Ed., “Topics in Fluorescence Spectroscopy Series,” Vol. 1-8. Plenum Press, New York (1992-2004).

4. Wolfbeis, O.S., Ed., "Springer Series on Fluorescence, Methods and Applications," Vol. 1-3, Springer, Berlin (2001-2004).

5. Mason, W.T., "Fluorescent and Luminescent Probes for Biological Activity," 2nd edn. Academic Press, San Diego (1999).

6. Haugland, R.P., "Handbook of Fluorescent Probes and Research Products," 10th edition. Molecular Probes, Eugene, OR, USA (2005). 
7. ASTM E 388-04, "Standard Test Method for Spectral Bandwidth and Wavelength Accuracy of Fluorescence Spectrometers," In: Annual book of ASTM standards, vol 03.06 (2004, original version 1972 ).

8. ASTM E 578 -07, "Standard Test Method for Linearity of Fluorescence Measuring Systems,” In: Annual book of ASTM standards, vol 03.06 (2007, original version 1983). 9. ASTM E 579 -04, "Standard Test Method for Limit of Detection of Fluorescence of Quinine Sulfate,” In: Annual book of ASTM standards, vol 03.06 (2004, original version 1984).

10. Certificate of Analysis, Standard Reference Material ${ }^{\circledR} 2940$ : Relative Intensity Correction Standard for Fluorescence Spectroscopy: Orange Emission, National Institute of Standards and Technology (2007). (https://srmors.nist.gov/ view_detail.cfm?srm=2940)

11. Certificate of Analysis, Standard Reference Material ${ }^{\circledR}$ 2941: Relative Intensity Correction Standard for Fluorescence Spectroscopy: Green Emission, National Institute of Standards and Technology (2007). (https://srmors.nist.gov/ view_detail.cfm?srm=2941)

12. Certificate of analysis, Standard Reference Material 936a, quinine sulfate dihydrate. National Institute of Standards and Technology (1994). (https://srmors.nist.gov/view_detail.cfm? srm=936A)

13. Certificate of analysis, Certified reference materials BAM-F001 - BAM-F005, Calibration Kit,Spectral Fluorescence Standards Federal Institute for Materials Research and Testing (BAM) (2006). These are available from BAM or from Sigma-Aldrich. 14. Certificate of Analysis, Standard Reference Material ${ }^{\circledR}$ 1932: Fluorescein Solution, National Institute of Standards and Technology (2003). (https://srmors.nist.gov/view_detail.cfm?srm=1932)

15. DeRose, P.C., Resch-Genger, U., Wang, L., Gaigalas, A.K., Kramer, G.W., Panne, U., "Need for and Metrological Approaches towards Standardization of Fluorescence Measurements from the View of National Metrology Institutes," in Fluorescence Standardization, Vol. 5 of Springer Series on Fluorescence (Springer-Verlag GmbH, Berlin Heidelberg), in press.

16. DeRose, P.C., "Standard Guide to Fluorescence - Instrument Calibration and Validation,” NISTIR 7458, National Institute of Standards and Technology (2007). 17. Marti, G.E., Vogt, R.F., Gaigalas, A.K., Hixson, C.S., Hoffman, R.A., Lenkei, R., Magruder, L.E., Purvis, N.B., Schwartz, A., Shapiro, H.M., Waggoner, A., "Fluorescence Calibration and Quantitative Measurements of Fluorescence Intensity, Approved Guideline,” NCCLS, I/LA24-A, Vol. 24 No. 26 (2004).

18. DeRose, P.C., Early, E.A., Kramer, G.W., "Qualification of a Fluorescence Spectrometer for Measuring True Fluorescence Spectra,” Rev.Sci.Instru., 78, 033107 (2007).

19. a) Hollandt, J., Taubert,R.D., Seidel, J., Resch-Genger, U., Gugg-Helminger, A., Pfeifer, D., Monte, C. and Pilz, W., "Traceability in Fluorometry-Part I: Physical Standards,” J.Fluoresc., 15, 301 (2005). b) Resch-Genger, U., Pfeifer, D., Monte, C., Pilz, W., Hoffmann, A., Spieles, M., Rurack, K., Hollandt, J., Taubert,D., Schonenberger, B. and Nording, P., "Traceability in Fluorometry-Part II: Spectral Fluorescence Standards,” J.Fluoresc., 15, 315 (2005). 
20. Hofstraat, J.W. and Latuhihin, M.J., “Correction of Fluorescence Spectra,”Appl. Spec., 48, 436 (1994).

21. Costa, L.F., Mielenz, K.D. and Grum, F., Chapter 4, "Correction of Emission Spectra," in Optical Radiation Measurements, Vol. 3, Measurement of

Photoluminescence, Mielenz, K.D., Ed., Academic Press, New York, p. 139-174 (1982).

22. Melhuish, W.H., Chapter 3, "Correction of Excitation Spectra," in Optical Radiation

Measurements, Vol. 3, Measurement of Photoluminescence, Mielenz, K.D., Ed., Academic Press, New York, p. 115-138 (1982).

23. Roberts, G.C.K., Chapter 7, "Correction of Excitation and Emission Spectra," in Techniques in Visible and Ultraviolet Spectrometry, Vol. 2, Standards in Fluorescence Spectrometry, Miller, J.N., Ed., Chapman and Hall, New York, p. 49-67 (1981).

24. DeRose, P.C., Smith, M.V., Mielenz, K.D., Blackburn, D.H. and Kramer, G.W., "Characterization of Standard Reference Materials 2941, Uranyl-Ion-Doped Glass, Spectral Correction Standard for Fluorescence,” J.Luminescence, 128, 257 (2008).

25. Melhuish, W.H., Chapter 5, "Sample-Induced Errors in Fluorescence Spectrometry," in Optical Radiation Measurements, Vol. 3, Measurement of Photoluminescence, Mielenz, K.D., Ed., Academic Press, New York, p. 175-193 (1982).

26. a) Lloyd, J.B.F., Chapter 5, "Inner Filter Effects, Sample Cells and their Geometry in Fluorescence Spectrometry," in Techniques in Visible and Ultraviolet Spectrometry, Vol. 2, Standards in Fluorescence Spectrometry, Miller, J.N., Ed., Chapman and Hall, New York, p. 27-43 (1981). b) West, M.A., Chapter 6, "Temperature Effects and Photodecomposition in Fluorescence Spectrometry," in Techniques in Visible and Ultraviolet Spectrometry, Vol. 2, Standards in Fluorescence Spectrometry, Miller, J.N., Ed. (Chapman and Hall, New York, p. 44-48 (1981).

27. Schwartz, A., Gaigalas, A.K., Wang, L., Marti, G.E., Vogt, R.F., Fernandez-Repollet, E., "Formalization of the MESF Unit of Fluorescence Intensity," Cytometry, 57B, 1 (2004).

28. Wang, L., Gaigalas, A.K., Abbasi, F., Marti, G.E., Vogt, R.F., Schwartz, A., "Quantitating Fluorescence Intensity from Fluorophores: Practical Use of MESF Values," J. Res. Natl. Inst. Stand. Technol., 107, 339 (2002).

29. Schwartz, A., Wang, L., Early, E., Gaigalas, A.K., Zhang, Y-z., Marti, G.E., Vogt, R.F., "Quantitating Fluorescence Intensity from Fluorophores: The Definition of MESF Assignment,” J. Res. Natl. Inst. Stand. Technol., 107, 83 (2002).

30. Mielenz, K.D., Eckerle, K.L., "Spectrophotometer Linearity Testing Using the Double-Aperture Method,” Appl.Opt., 11, 2294 (1972).

31. Zwinkels, J.C., Gignac, D.S. "Automated High Precision Variable Aperture for Spectrophotometer Linearity Testing,” Appl. Opt., 30, 1678 (1991).

32. Gardecki, J.A., Maroncelli, M., "Set of Secondary Emission Standards for Calibration of the Spectral Responsivity in Emission Spectroscopy,” Appl. Spectr., 52, 1179 (1998).

33. Velapoldi, R.A., Tonnesen, H.H., "Corrected Emission Spectra and Quantum Yields for a Series of Fluorescent Compounds in the Visible Spectral Region, J. Fluoresc., 14, 465 (2004).

34. Holbrook, R.D., DeRose, P.C., Leigh, S.D., Rukhin, A.L., Heckert, N.A., "ExcitationEmission Matrix Fluorescence Spectroscopy for Natural Organic Matter

Characterization: A Quantitative Evaluation of Calibration and Spectral Correction Procedures”, Appl.Spec., 60, 791 (2006). 
35. Demas, J.N. "Measurement of Photon Yields," in Optical Radiation Measurements, Volume 3, Measurement of Photoluminescence, Mielenz, K.D., Ed., Academic Press, New York (1982) pp 228-233.

36. Lakowicz, J.R., "Principles of Fluorescence Spectroscopy", second edition, Kluwer Academic/Plenum Publishers, New York (1999) pp.49-52

37. Kovach, R.J., Peterson, W.M., "The Measurement of Sensitivity in Fluorescence Spectroscopy,” Am. Lab., 32G, (1994).

38. Froehlich, P., "Under the sensitivity specification for a fluorescence spectrophotometer," Int. Lab., 42, (1989).

39. "Technical Note: The Measurement of Sensitivity in Fluorescence Spectroscopy," Photon Technology International (1997-1999). http://www.pti-nj.com/tech_3.html

40. Lakowicz, J.R., "Principles of Fluorescence Spectroscopy", second edition, Kluwer Academic/Plenum Publishers, New York (1999) pp.95-140, 141-184.

41. Boens, N., Ameloot, M., et.al., "Fluorescence Lifetime Standards for Time and Frequency Domain Fluorescence Spectroscopy,” Anal.Chem., 79, 2137 (2007).

42. Lakowicz, J.R., "Principles of Fluorescence Spectroscopy", second edition, Kluwer Academic/Plenum Publishers, New York (1999) pp.645-651.

43. Certain commercial equipment, instruments, or materials are identified in this paper to foster understanding. Such identification does not imply recommendation or endorsement by the National Institute of Standards and Technology, nor does it imply that the materials or equipment identified are necessarily the best available for the purpose.

44. ASTM E 131-02, "Standard Terminology Relating to Molecular Spectroscopy,” In: Annual book of ASTM standards, vol 03.06 (2002).

45. Braslavsky, S.E., "Glossary of Terms Used in Photochemistry, 3rd Edition, (IUPAC Recommendations 2006),” Pure Appl.Chem., 79, 293 (2007).

46. Larason, T.C., Bruce, S.S., Parr, A.C., Natl. Bur. Stand. (U.S.) Spec. Publ. 250-41, U.S. GPO, Washington, D.C. (1998).

47. Walker, J.H., Saunders, R.D., Jackson, J.K., McSparron, D.A., Natl. Bur. Stand.

(U.S.) Spec. Publ. 250-20, (U.S. GPO, Washington, D.C., 1987).

48. J. H. Walker, R. D. Saunders and A. T. Hattenburg, Natl. Bur. Stand. (U.S.) Spec. Publ. 250-1, U.S. GPO, Washington, D.C. (1987).

49. Weidner, V.R., Hsia, J.J., Natl. Bur. Stand. (U.S.) Spec. Publ. 250-6, U.S. GPO, Washington, D.C. (1987).

50. Palmer, C., "Diffraction Grating Handbook,” fourth edition, Richardson Grating Laboratory, Rochester, N.Y. (2000).

51. Yappert, M.C., Ingle, Jr., J.D., Appl.Spec. 43, 759 (1989).

52. Parker, C.A., Barnes, W.J., Analyst 82, 606 (1957).

53. Chalmers, J., Griffiths, P., Eds., "Handbook of Vibrational Spectroscopy,” John Wiley \& Sons, Ltd, New York (2002). 